\title{
Feedforward control strategies of subjects with transradial amputation in planar reaching
}

\author{
Anthony J. Metzger, MBE; ${ }^{1-2}$ Alexander W. Dromerick, MD; ${ }^{3-5}$ Christopher N. Schabowsky, MS; ${ }^{1-2}$ Rahsaan \\ J. Holley, MS; ${ }^{4}$ Brian Monroe, BS; ${ }^{6}$ Peter S. Lum, PhD ${ }^{1-3^{*}}$ \\ ${ }^{1}$ Center for Applied Biomechanics and Rehabilitation Research, National Rehabilitation Hospital, Washington, DC; \\ ${ }^{2}$ Department of Biomedical Engineering, Catholic University of America, Washington, DC; ${ }^{3}$ Department of Veterans \\ Affairs Medical Center, Washington, DC; ${ }^{4}$ Neuroscience Research Center, National Rehabilitation Hospital, Washing- \\ ton, DC; ${ }^{5}$ Departments of Rehabilitation Medicine and Neurology, Georgetown University, Washington, DC; ${ }^{6}$ District \\ Amputee Care Center, Washington, DC
}

\begin{abstract}
The rate of upper-limb amputations is increasing, and the rejection rate of prosthetic devices remains high. People with upper-limb amputation do not fully incorporate prosthetic devices into their activities of daily living. By understanding the reaching behaviors of prosthesis users, researchers can alter prosthetic devices and develop training protocols to improve the acceptance of prosthetic limbs. By observing the reaching characteristics of the nondisabled arms of people with amputation, we can begin to understand how the brain alters its motor commands after amputation. We asked subjects to perform rapid reaching movements to two targets with and without visual feedback. Subjects performed the tasks with both their prosthetic and nondisabled arms. We calculated endpoint error, trajectory error, and variability and compared them with those of nondisabled control subjects. We found no significant abnormalities in the prosthetic limb. However, we found an abnormal leftward trajectory error (in right arms) in the nondisabled arm of prosthetic users in the vision condition. In the no-vision condition, the nondisabled arm displayed abnormal leftward endpoint errors and abnormally higher endpoint variability. In the vision condition, peak velocity was lower and movement duration was longer in both arms of subjects with amputation. These abnormalities may reflect the cortical reorganization associated with limb loss.
\end{abstract}

Key words: amputation, feedforward, motor control, prosthesis, reaching, training, trajectory, transradial, upper limb, velocity.

\section{INTRODUCTION}

The prevalence of individuals living in the United States with upper-limb amputations is approximately 41,000 , with a projected increase of 131 percent by the year 2050 [1]. Although prosthetic devices are prescribed, 38 to 50 percent of people with upper-limb amputation fitted for a prosthetic device fail to use it regularly or abandon it altogether [2-3]. Intensive training in prosthetic device use has been associated with increases in upper-limb performance [4]. By understanding the movement strategies of prosthetic device wearers, researchers can alter prosthetic devices and develop training protocols to improve prosthetic device use and acceptance.

Reaching to a target is one of the fundamental arm tasks. Goal-directed reaching features feedforward and feedback control systems. Feedforward control produces

Abbreviations: $\mathrm{ADL}=$ activity of daily living, ANOVA $=$ analysis of variance, CNS = central nervous system.

*Address all correspondence to Peter S. Lum, PhD; Department of Biomedical Engineering, Catholic University of America, Pangborn Hall, Room 131, 620 Michigan Ave NE, Washington, DC 20064; 202-319-5657; fax: 202319-4287. Email: lum@cua.edu

DOI:10.1682/JRRD.2009.06.0075 
predictive motor commands that reflect previous reaching history, and feedback control is the ability to make adjustments during the movement based on error detection by the visual and proprioceptive sensory systems [5-7]. By eliminating visual feedback, individuals must rely more on the feedforward model to achieve the target accurately. In nondisabled subjects, when vision of the limb is occluded, reaching results in larger endpoint errors [8]. Thus, a paradigm of rapid vision-occluded movements allows close examination of the feedforward control system.

Feedforward systems rely on the formation of an internal model [9]. The internal model of the arm accounts for the weight, length, and distribution of mass in each arm segment. The feedforward control system uses the internal model of the arm to develop a plan for any movement given the desired endpoint, starting point, speed, and expected perturbations. Previous work has shown that people with transradial amputation can develop an accurate internal model of curl field perturbations during repetitive reaching movements [10]. If we can detect specific deficits in the internal models of prosthetic limbs, training protocols can be developed that focus on improving these models, potentially improving prosthetic device function and acceptance.

We examined the accuracy of ballistic reaching in subjects with unilateral transradial amputation. The use of prosthetic devices alters the inertial properties of the limb, which may hinder the formation of an accurate internal model of the arm. In order for prosthetic device users to perform accurate reaching movements with their prosthetic arm, they must formulate a new internal model of that arm that accounts for the new biomechanical properties of the limb. We tested both the prosthetic and nondis- abled arms with and without visual feedback and compared them with age-matched, dominance-matched nondisabled control subjects. We hypothesized that subjects with amputation would exhibit abnormal reaching patterns because of inaccurate feedforward control.

\section{METHODS}

Ten unilateral transradial upper-limb prosthetic device users, aged $40 \pm 4$ years (all data presented as mean \pm standard error unless otherwise noted) and ten nondisabled control subjects, aged $37 \pm 4$ years, participated in the study. The Table summarizes the prosthetic device users' clinical data. We compared the data collected from the prosthetic arm, which was always tested first, with the first arm tested from the control subjects and the nondisabled arm with the second arm tested from the control subjects. For naming purposes, "prosthetic arm" will represent the prosthetic device attached to the residual limb of the subjects with amputation, whereas "nondisabled arm" will represent the contralateral arm. Of the subjects with amputation, six had right-handed prosthetic devices and four had left-handed prosthetic devices; therefore, we tested six control subjects' right arms first and four control subjects' left arms first. All subjects were right-hand dominant (preinjury state for subjects with amputation). We screened subjects for neurological and orthopedic injuries, such as hemiparesis, dementia, schizophrenia, and motor disorders, which would have disqualified them from the study.

To confirm that the inertial properties of the prosthetic arm were different from a nondisabled limb, we

Table.

Prosthetic device group clinical summary.

\begin{tabular}{cccccc}
\hline Subject & Age (yr) & Chronicity (yr) & Nine Hole Peg Test (s) & Prosthesis Type & Amputated Arm \\
\hline 1 & 46 & 2.0 & 118 & Body powered: Hook \\
2 & 31 & 1.2 & $300^{*}$ & Body powered: Hook & Boft \\
3 & 51 & 50.0 & 103 & Myoelectric: Hand & Left \\
4 & 26 & 1.8 & $300^{*}$ & Myoelectric: Hand & Right \\
5 & 37 & 30.0 & 207 & Body Powered: Hook & Right \\
6 & 36 & 2.8 & 109 & Body powered: Hook & Body powered: Hook \\
7 & 42 & 5.6 & 80 & Myoelectric: Hook & Right \\
8 & 67 & 45.0 & 45 & Myoelectric: Hand & Right \\
9 & 31 & 12.4 & NA & & Left \\
\hline
\end{tabular}

${ }^{*}$ Maximum value for Nine Hole Peg test is 300 seconds (5 minutes).

$\mathrm{NA}=$ information not available. 
measured the mass, center of mass, and moment of inertia (pendulum method) of a typical body-powered prosthesis (stainless steel hook) and myoelectric prosthesis and compared these values with tabulated values for the forearm and hand of a $180 \mathrm{lb}$ male with forearm length that matched the forearm length of the prosthesis [11]. The center of mass of the body-powered prosthesis (measured relative to the elbow location when the prosthesis was being worn) was nearly identical to that of the human forearm and hand. However, the mass and moment of inertia of the prosthesis were only 49 and 39 percent of a nondisabled human limb, respectively. The center of mass of the myoelectric prosthesis was $1.3 \mathrm{~cm}$ more distal than a human forearm/hand. The mass and moment of inertia of the myoelectric device were 57 and 39 percent of a nondisabled human upper limb, respectively.

Subjects interacted with a robot (InMotion2 ShoulderElbow Robot, Interactive Motion Technologies, Inc; Watertown, Massachusetts) with a handle located at the end effector (Figure 1). This robot allows reaches in a horizontal plane, has two programmable motors that control the position of the robot links, and has two encoders that measure the position of each link. We used two software programs (MATLAB 7.1 and XPCtarget 2.8, The MathWorks; Natick, Massachusetts) to control the robot and collect position data from the encoders. To decrease the intrinsic inertial anisotropy (unequal inertial properties along different directions) of the robot and compensate for inertia of the linkage system, we implemented algorithms based on an inverse dynamic model of the robot. Starting from free-body diagrams of the four robot links, we derived the dynamic equations of motion for the robot. Link mechanical properties were available from Interac- tive Motion Technologies, Inc. We obtained angular velocities and accelerations from real-time digital differentiation of the encoder signals. At each time sample, we used the kinematic data to calculate the torques at the motors that were needed to drive the motion at that instant in time. These torque commands were then sent to the motors in real-time, thereby partially canceling out the acceleration and velocity dependent forces felt at the handle. Performance of this compensation algorithm was limited by the accuracy of real-time digital differentiation algorithms. Thus, the robot measures the kinematics of the reach while providing minimal resistance to the movements. Peak resistance forces for the two movement directions tested differed by less than $3 \mathrm{~N}(6.6 \mathrm{~N}$ vs $4.1 \mathrm{~N})$, and the lateral forces never exceeded $1 \mathrm{~N}(0.7 \mathrm{~N}$ vs $0.8 \mathrm{~N})$ in any movement direction. A $t$-test on lateral forces indicated no significant differences between directions ( $p=$ 0.07). Furthermore, we always performed group comparisons between groups that had equal ratios of left and right arms, so that any possible effects of robot anisotropy were minimized.

We seated subjects in an adjustable chair and raised them up to the robotic arm so that the plane of the reaching movements was slightly below shoulder height. We placed the subjects' forearms in a splint attached to the robot to restrict wrist rotation. The prosthetic device subjects placed their forearms in the splint with their terminal device passively wrapped around the handle. The wrist splint also supported the arm against gravity.

Subjects performed outward reaches to two alternating targets, returning to the original starting point between reaches. A computer monitor displayed handle position and target location in real time in front of the subject. The (a)

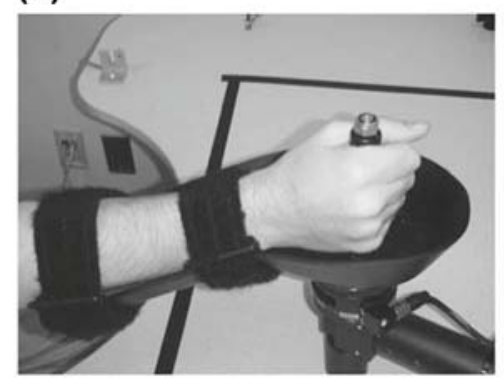

(b)

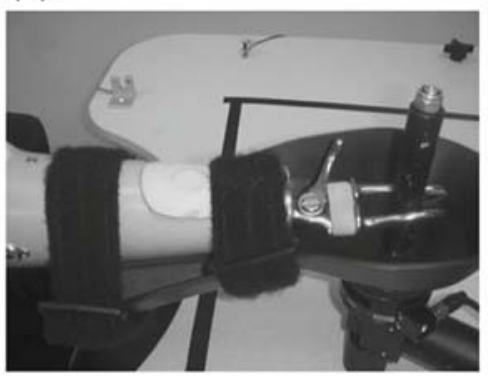

(c)

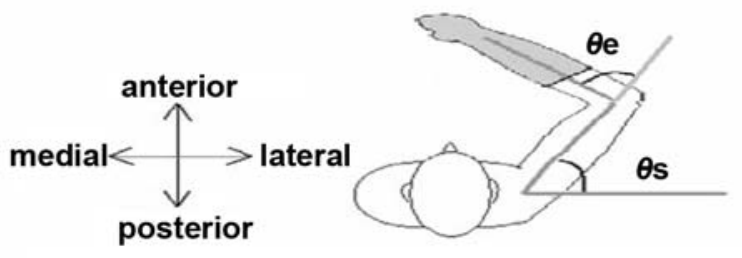

Figure 1.

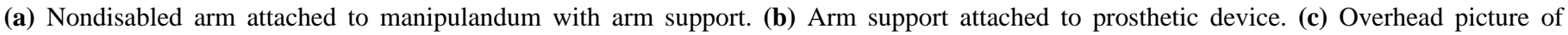

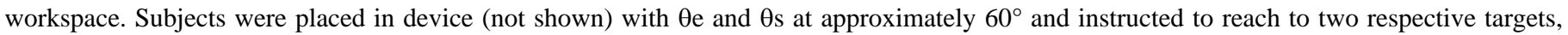
contralateral (CT) and ipsilateral (IT). 
starting position was displayed as a $1 \mathrm{~cm}$ circle in the midline of the subject, $15 \mathrm{~cm}$ from the subject's body. The targets were presented as $1 \mathrm{~cm}$ circles positioned at $45^{\circ}$ and $135^{\circ}$ from the medial-lateral axis and $15 \mathrm{~cm}$ from the starting position. We instructed the subjects to perform quick, ballistic movements to the targets. We only analyzed the outward reaches to the targets. The desired range of peak tangential velocity was 45 to $55 \mathrm{~cm} / \mathrm{s}$, and we provided visual feedback after each reach to encourage the subject to maintain a consistent velocity. If the subject's reach was too slow or too fast, the starting position circle would turn green or red, respectively. If the subject reached within the desirable velocity range, the starting circle would turn white.

The subjects performed two conditions before repeating the process for the other arm. The first condition consisted of 20 reaches (10 per target) with vision, in which subjects observed handle position in real time. After the subject moved the handle into the start circle and velocity dropped to zero, the start circle disappeared and the target circle appeared. We instructed the subject to move into the target with the proper speed. After the subject achieved the target and velocity dropped to zero, the target circle disappeared and the start circle reappeared. The second condition consisted of 40 reaches (20 per target) with vision occlusion, in which the handle position disappeared after the start circle was achieved and as the target appeared. We told the subject to reach to the target without visual feedback of handle position. The handle display reappeared after the subject stopped his or her reach, so that he or she could finish the reach and return to the starting position. We placed a wooden canopy over the robot's linkage system to occlude the vision of the actual handle and the subject's arm.

We calculated handle position and tangential velocity. We defined initiation and cessation of each outward reach as 5 percent of maximum tangential velocity. We defined movement duration as time from initiation to cessation. We also obtained the time between initiation and peak velocity (acceleration time) for comparison. Accuracy was based on three factors: endpoint error, peak error, and endpoint variability. We divided endpoint error into two components: aiming error and distance error, representing directional (left/right) error and overshoot/undershoot error, respectively. Figure 2 illustrates the sign designation of these errors for a trial. All results and figures will be represented as right-handed movements; we flipped left-handed data about the midline before analyzing it. We defined peak error as the maximum orthogonal distance between the actual trajectory and the "ideal" straight trajectory between the starting point and endpoint of each reach. We defined variable error as the average distance between endpoint locations of each reach and the average endpoint location for all reaches to a particular target. This measure gives a value representing the variability of the subjects' reach endpoints.

We averaged outcome measures across trials and analyzed them with repeated-measures analysis of variance (ANOVA). The main comparisons were between the prosthetic limb and the first tested limb of control subjects and between the nondisabled limb of subjects with amputation and the second tested limb of control subjects. The between-subject factor was group (prosthetic, control) and the within-subject factor was target. We compared peak velocities between prosthetic subjects and control subjects using $t$-test analysis. We performed Pearson correlation analysis comparing the outcome measures and patient information, such as age, chronicity, Nine Hole Peg Test results, and Action Research Arm Test results. The Nine Hole Peg Test is a standardized clinical assessment of arm motor control, measured as the time required to complete the task [12]. The Action Research Arm Test is a standardized clinical assessment of arm movement divided into four subtests: grasp, grip, pinch, and gross movement [13]. To test for limb dominance or testing order effects, we performed repeated-measures ANOVA on control subject data (within-subject factors of target and side; between-subjects factors of testing order). To examine the possibility of a learning effect, we compared the mean of the first five trials for each subject

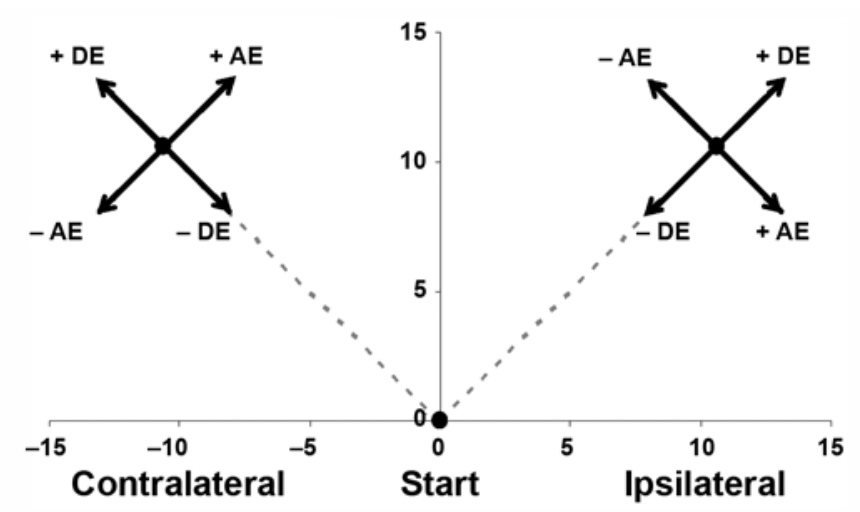

Figure 2.

Sign convention for distance error (DE) and aiming error (AE). 
with the mean of the last five trials on all outcome measures in a repeated measure ANOVA with within-subject factors of target and trial.

\section{RESULTS}

\section{Peak Reaching Velocities and Movement Durations}

Without visual guidance, we found no significant differences in peak velocity between groups $(p>0.26)$. However, with visual feedback, the subjects with amputation had difficulties maintaining the specified velocity range. With their prosthetic arms, the subjects reached with an average maximum velocity of $39.3 \pm 2.3 \mathrm{~cm} / \mathrm{s}$ compared with the control subjects' average peak velocity of $45.6 \pm$ $1.9 \mathrm{~cm} / \mathrm{s}(p=0.05)$. We found similar results with the nondisabled arms, which had an average maximum velocity of $39.5 \pm 2.2 \mathrm{~cm} / \mathrm{s}$ compared with $47.5 \pm 1.4 \mathrm{~cm} / \mathrm{s}$ for control subjects $(p=0.01)$. We calculated Pearson correlation coefficients to determine if velocity was significantly correlated with our outcome measures. Correlations between our outcome measures (aiming, distance, peak, and variable error) and peak velocity were not significant ( $p>$ 0.01 ). Thus, our outcome measures were not significantly influenced by the velocity difference between both groups in the visual feedback condition.

During the trials with visual guidance, the subjects with amputation had significantly longer movement durations in their prosthetic arm $(p=0.02)$ and in their nondisabled arm $(p=0.01)$ than control subjects. When subjects performed reaches without visual guidance, we observed abnormally long movement durations in the nondisabled arms $(p=0.05)$, but not the prosthetic arms $(p=0.07)$. Analysis of the acceleration times illustrated no significant differences between groups in any of the conditions $(p>0.08)$.

\section{Accuracy of Reaching with Visual Guidance}

The vision condition produced no significant differences between reaching with prosthetic arms and control subjects' arms (see Figure 3 for average trajectories). Repeated-measures ANOVA found that prosthetic arms were not different from control subjects' arms in aiming error $(p=0.82)$, distance error $(p=0.19)$, variable error $(p=0.96)$, or peak error $(p=0.37)$.

The nondisabled arm of subjects with amputation reached with an abnormal leftward trajectory in the vision condition (see Figure 4 for average trajectories).

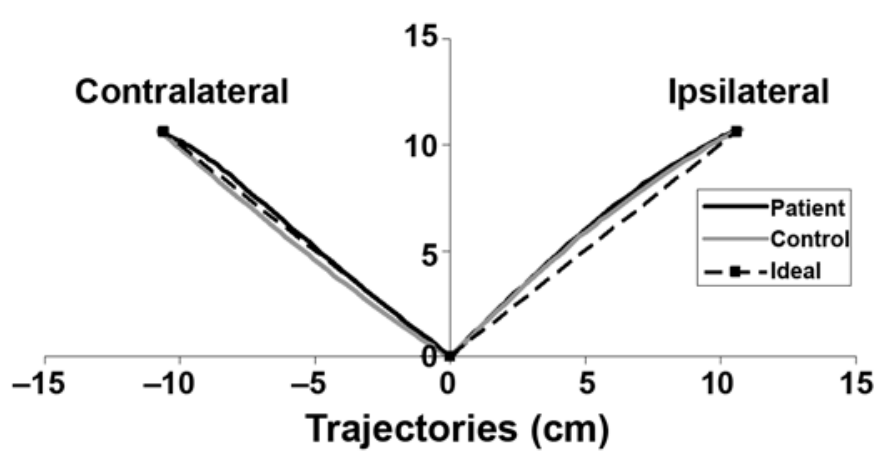

Figure 3.

Average reach trajectories of prosthetic arm during condition with visual feedback. Graphs reflect right-handed movements.

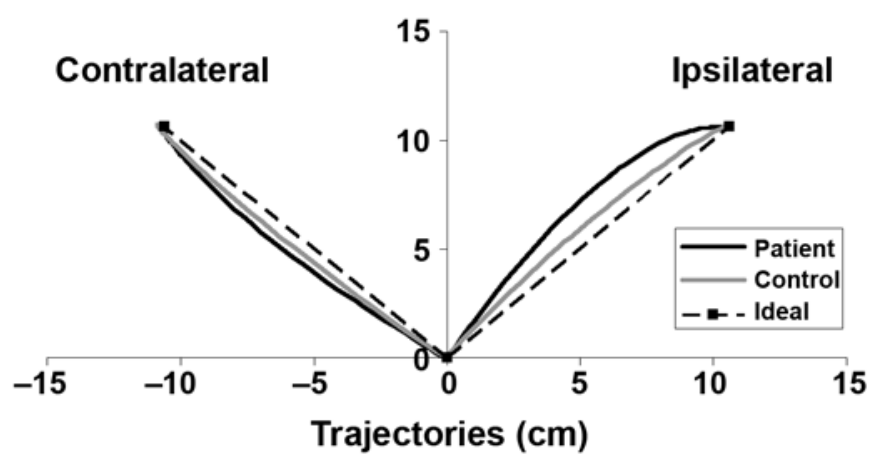

Figure 4.

Average reach trajectories of nonprosthetic arm during condition with visual feedback. Graphs reflect right-handed movements.

ANOVA revealed a significant difference in peak error between groups $(p=0.01)$. Post hoc analysis found group differences of $1.07 \mathrm{~cm}(p=0.02)$ and $0.37 \mathrm{~cm}(p=0.01)$ for ipsilateral and contralateral targets, respectively, indicating that for both targets the nondisabled arms reached with a more leftward trajectory than control subjects' arms, and this effect was significant for the ipsilateral trajectory. Variable error $(p=0.79)$ and aiming error $(p=$ 0.33 ) were not significantly different between groups. The ANOVA also revealed a significant group difference in distance error $(p=0.01)$, with the prosthetic group completing their reach closer to the target; however, the mean difference was less than $3 \mathrm{~mm}$.

\section{Accuracy of Reaching Without Visual Guidance}

No significant differences were displayed in the vision occlusion condition between prosthetic arms and 
control subjects' arms (see Figure 5 for average trajectories). ANOVA of the prosthetic arms revealed no significant difference from control subjects in distance error $(p=0.60)$, aiming error $(p=0.14)$, peak error $(p=0.42)$, or endpoint variability ( $p=0.51)$.

When we eliminated visual feedback, we found performance of the nondisabled arm again significantly worse than arms of the control subjects (see Figure 6 for average trajectories). The distance errors displayed no significant group differences ( $p=0.92$ ), but we found a large group difference in aiming error $(p=0.01)$. We also found a significant interaction effect between the subject group and target $(p=0.03)$, indicating that the group differences in aiming error were different for the two targets. The nondisabled arm of the subjects with amputation had a large leftward aiming error for the ipsilateral

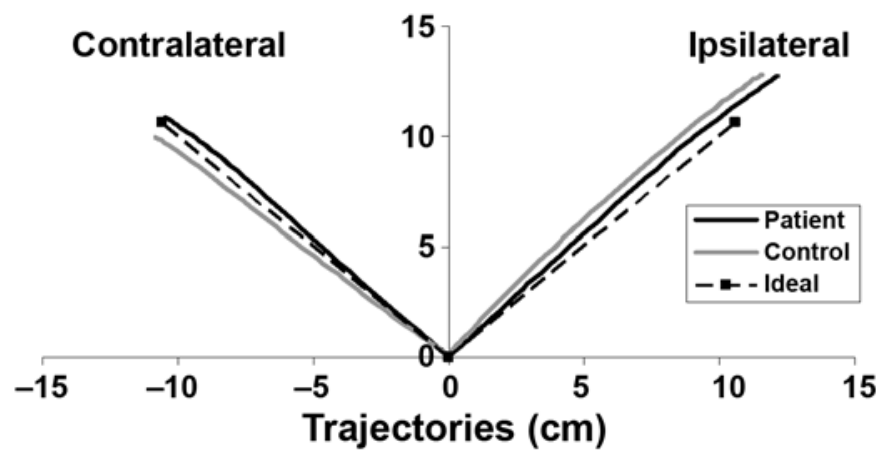

Figure 5.

Average reach trajectories of prosthetic arm during condition without visual feedback. Graphs reflect right-handed movements.

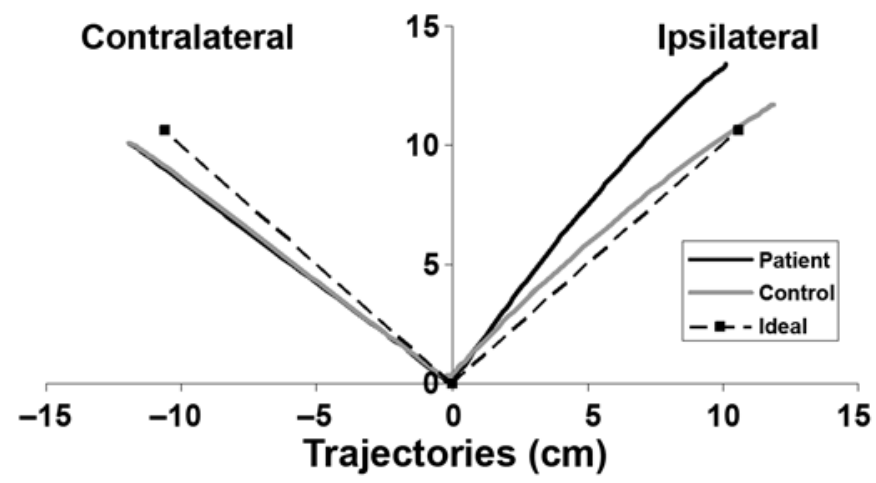

Figure 6.

Average reach trajectories of nonprosthetic arm during condition without visual feedback. Graphs reflect right-handed movements. target of $2.33 \mathrm{~cm}$ versus $0.11 \mathrm{~cm}$ rightward error for control subjects $(p=0.01)$. This group difference was not present in the contralateral target $(p=0.92)$.

Under the vision occlusion condition, the reach endpoints of the prosthetic group's nondisabled arm were not only less accurate but also were more variable compared with those of the control subjects. Repeated-measures ANOVA found that the nondisabled arms of subjects with amputation had larger endpoint variability than control subjects' arms $(p=0.02)$. Reaches using the nondisabled arm had an average variable error of $2.05 \pm$ $0.16 \mathrm{~cm}$ for the contralateral target and $2.51 \pm 0.18 \mathrm{~cm}$ for the ipsilateral target, while the control subjects had values of $1.56 \pm 0.15 \mathrm{~cm}$ and $2.06 \pm 0.17 \mathrm{~cm}$, respectively. The peak errors of the subjects with amputation were not significantly different from control subjects ( $p=$ 0.93). In conclusion, without visual feedback, nondisabled arms of subjects with amputation had abnormal leftward endpoint errors and higher variability than control subjects.

\section{Correlation Analysis}

Pearson correlation coefficients determined that age $(p>0.32)$ and chronicity $(p>0.09)$ were not significantly correlated with the outcome measures. We found similar results when comparing the outcome measures with clinical test results: Nine Hole Peg Test $(p>0.09)$ and Action Research Arm Test $(p>0.17)$. ANOVA comparing myoelectric versus body-powered prosthetic devices did not reveal any significant differences for any of the outcome measures $(p>0.39)$.

\section{Limb Dominance, Order, and Learning Effects}

Repeated-measures ANOVA on the control data determined that in the vision occlusion condition, dominance was a significant factor in aiming error $(p=0.02)$, with group means of 0.25 and $0.81 \mathrm{~cm}$ for the right and left arms, respectively. It was also revealed that order of testing was significantly related to the variable error ( $p=$ 0.01 ). The second tested limb had a variable error of $1.80 \mathrm{~cm}$, as opposed to $2.17 \mathrm{~cm}$ for the first tested limb. We found no other dominance or order effects in control subjects. It was not possible to test for order effects in the subjects with amputation because we always tested the prosthetic limb first. When examining the data of subjects with amputation, we found no significant dominance effects in either limb for any of our outcome measures $(p>0.3)$. When comparing early reaches with late reaches 
to examine learning effects, we observed no significant improvements in any of the outcome measures in both the vision and vision occlusion conditions ( $p>0.08$ ).

\section{DISCUSSION}

Our purpose was to investigate kinematic errors during goal-directed reaching performed by both arms of subjects with unilateral amputation; however, we only observed decreased accuracy in the nondisabled arms and not the prosthetic arms. In the prosthetic arm, the elimination of visual feedback caused larger endpoint errors, but we found similar errors in control subjects. Therefore, the abnormal mechanical properties of the prosthetic device did not increase feedforward control errors. The combination of a sufficient internal model of the arm and the residual proprioceptive receptors in the elbow and shoulder were adequate for normal accuracy in planar reaching. However, peak velocity was lower and movement duration was longer in both arms of subjects with amputation than in control subjects, especially in the vision condition.

Since we found no significant accuracy abnormalities in the prosthetic arm for this experiment, the patients must have adapted to their prosthetic device using appropriate compensatory strategies. Despite the altered mechanical properties of the prosthetic arm, the subjects with amputation were able to produce the proper torques about the shoulder and elbow to move their prosthetic limbs accurately toward the targets. Since the ballistic movements used in this study require feedforward control to specify initial movement parameters [14], the internal model of the prosthetic arm must have adapted to the mechanical properties of the prosthetic device. This ability to adapt may be related to the motor adaptation displayed by nondisabled arms when placed in a distorted "curl-field" environment $[9,15]$, in which the brain is able to incorporate sensory feedback obtained during reaching to update the internal model of the environment. In a previous study, we demonstrated that prosthetic arms can also adapt to curlfield environments, although not as well as nondisabled control subjects [10]. This implies that subjects with amputation are able to use the residual sensory feedback to overcome the new mechanical properties of the arm and properly formulate a new internal model. This is consistent with the concept of extended physiological proprioception introduced by Simpson [16]. He compared the use of a prosthetic device with a golfer swinging a club, whereby the central nervous system (CNS) incorporates foreign objects into the internal model of the arm. These findings indicate that in subjects with below-elbow amputation, the basic CNS control mechanisms for reaching are still in place and can adapt appropriately to the new mechanical properties of the prosthetic arm. However, it is unlikely that similar results will be found in subjects with higher-level amputation where control of the elbow will be through a nonnative mechanism. For example, subjects with transhumeral amputation using a body-powered elbow showed good performance when moving the elbow to a memorized angle, but performance degraded when subjects were asked to perform dynamic pointing and tracking tasks [17].

However, the nondisabled arms of subjects with amputation demonstrated abnormal behavior during the same reaching experiment. When reaching with visual feedback, the subjects with amputation tended to initiate their reach to the left of the ideal trajectory before performing a corrective movement toward the target. When we eliminated vision of the arm, the nondisabled arms of the subjects with amputation no longer made large corrective movements. Instead, they continued on an inaccurate trajectory resulting in an inaccurate endpoint skewed to the left. However, this was only significant for the ipsilateral target because the control subjects displayed similar behavior during reaches to the contralateral target. The subjects with amputation also displayed more variability in their endpoint locations than control subjects during the vision occlusion condition.

Several possible explanations exist for the abnormal performance of the nondisabled arm. The kinematics of the nondisabled arm might have been affected by the testing order of the two arms. We always tested the nondisabled arm immediately after the prosthetic arm, so the strategy used to control the nondisabled arm may have been affected by the strategy used to control the prosthetic limb. The feedforward motor commands required for the prosthetic arm were very different than those required for the nondisabled arm because of the different mechanical properties of the two limbs. While performing the movement toward the ipsilateral target, subjects initiated nondisabled arm trajectories in a direction medial to the ideal trajectory, indicating insufficient elbow extension and/or shoulder transverse extension. As noted previously, the prosthetic devices have lower mass and moment of inertia than an nondisabled forearm and would require less torque about the elbow and shoulder to perform a reach. The subjects with prosthetic arms may have used the reaching 
strategy of their prosthetic limb for their nondisabled limb. The larger mass and moment of inertia of the nondisabled limb would have required extra torque about the elbow and shoulder as compared with the prosthetic limb. A related finding was reported by Weeks et al. [18], who found that the nondisabled hand produced a stabilizing grip force similar to the prosthetic hand, both of which were significantly larger than that of controls. The subject used the same grip strategy across arms despite the anatomical differences. These observations might also be associated with previous findings regarding interlimb transfer of a novel motor task, in which exposure to a motor task by one arm can improve performance of the opposite arm [19-23]. In our study, the effect of the transfer may have decreased performance of the nondisabled arm in subjects with amputation because of differences in the mechanical properties of the two arms. However, if order was a major factor, one would have expected gradual correction of errors in the nondisabled arm over the course of repeated trials, since error feedback was available in all trials. We did not detect any improvement of performance over the repeated trials. We did detect an order effect in the vision occlusion condition in control subjects, but this was restricted to the variable error only: we saw no order effect in any of the other measures. This finding further argues against order as a major factor in this experiment.

The target dependency of errors in the nondisabled arm might be explained by contributions from the ipsilateral hemisphere to movement of the nondisabled arm. This argument is based on the hypothesis that an internal model of a joint cannot exist independent of the model of the contralateral homologous joint or that the CNS uses resources from both hemispheres to produce a single internal model that is used for the same joint on both sides of the body [24]. The lack of accuracy abnormalities in the prosthetic arm suggests that the contralateral hemisphere has adapted to the altered musculoskeletal properties of the prosthetic forearm. This adaptation may have compromised this hemisphere's role in movements of the ipsilateral nondisabled arm. This might explain why in the vision occlusion condition, we only observed deficits in the nondisabled arm with the ipsilateral target and not with the contralateral target. Inverse dynamic analyses of these movements have shown that movements to the contralateral target are driven primarily by shoulder transverse flexion [8]. Since the humerus and shoulder musculature in the affected arm were not injured, the internal model of this joint can remain similar to that of the nondisabled arm. Movement toward the ipsilateral target is primarily an elbow extension. The internal model of the elbow needs to be different between limbs to account for alterations in forearm mechanical properties and elbow musculature in the prosthetic arm. This disparity between limbs at the elbow, but not the shoulder, may explain the large deficits in the ipsilateral movements but not the contralateral movements.

The abnormal reaching of the nondisabled arm of subjects with amputation may be related to the ipsilesional deficits observed in patients with stroke. While the brains of subjects with amputation are nondisabled, significant reorganization occurs after the injury. After amputation, the human brain undergoes reorganization of the motor cortex in the areas previously designated to the missing limb [25-27]. It has been shown that areas previously delegated to the missing limb begin functioning with adjacent areas of the cortical map. The remaining body parts near the amputation level gain new cortical representations in the brain, which may affect motor function. This phenomenon also occurs in patients with stroke as other areas of the brain invade the affected area, resulting in cortical restructuring [28-29]. Similar to our results in the nondisabled arm of subjects with amputation, research has shown that patients with stroke also demonstrate motor deficits in their ipsilesional arm [25,30-33]. These deficits in the "unaffected" limb may be related to imbalanced interhemispheric inhibition caused by cortical reorganization in the motor cortex of both patients with stroke and subjects with amputation [34].

Our results may be related to recent research on brain lateralization, which implies that each hemisphere, based on dominance, is specialized for certain behavior [35-36]. The dominant hemisphere is specialized for feedforward planning, whereas the nondominant hemisphere is specialized for feedback corrections. Our data from control subjects support this hypothesis. In the vision occlusion condition, which is highly dependent on feedforward control, the right (dominant) arms had smaller aiming errors than the left (nondominant) arms. However, in the amputation group, both left and right nondisabled arms had large peak errors during visually guided movements and large aiming errors in the vision occlusion condition. Therefore, the traditional attributes of dominant/nondominant limbs may not apply to the population with amputation because of altered usage patterns.

The decreased peak velocity and increased movement duration in the prosthetic limb in the vision condition might be due to a number of factors. Dynamic torques increase with velocity of movement, and uncertainty in 
the internal limb model might lead to an adaptation of decreased speed to maintain accuracy. Alternatively, dynamic forces at the socket interface will increase with speed of movement, further motivating a choice to move with reduced speeds. Reduced muscle torque at the elbow in some of the subjects may also have been a factor. Interestingly, the parallel abnormalities in velocity and movement duration observed in the nondisabled arm of subjects with amputation further support the hypothesis that the CNS uses similar control strategies in both limbs.

\section{CONCLUSIONS}

Despite the lack of accuracy deficits in the prosthetic limb, we cannot make conclusions regarding complete function of the limb in everyday tasks. For gross motor reaching, the feedforward control of the prosthetic limb was shown to be sufficient, but other aspects of limb function (i.e., grasping) may require more visual feedback to compensate for feedforward control errors. To fully assess patient-prosthesis functionality in clinical applications, researchers must determine the efficiency of prosthetic users during activities of daily living (ADLs). ADLs involve distal manipulation and three-dimensional reaching components, which we did not address in this study but are major components of functionality. Distal manipulation would require the person to determine the appropriate configuration of the hook/hand and the extent to which to open and close the terminal device. Threedimensional reaching would incorporate gravity effects, which could hinder performance because of the altered mass properties of the device. Therefore, prosthetic users are able to adapt to their prosthesis in horizontal planar reaching, but additional research is required to determine efficiency in more complex reaching tasks. Additional studies of the nondisabled arm may also lead to improved functionality and will likely yield insights into the cortical control of arm movements.

\section{ACKNOWLEDGMENTS}

\section{Author Contributions:}

Study concept and design: A. W. Dromerick, C. N. Schabowsky, P. S. Lum.

Acquisition of data: A. J. Metzger, A. W. Dromerick, C. N. Schabowsky. Analysis and interpretation of data: A. J. Metzger, C. N. Schabowsky, P. S. Lum.
Drafting of manuscript: A. J. Metzger.

Critical revision of manuscript for important intellectual content:

A. J. Metzger, P. S. Lum.

Statistical analysis: A. J. Metzger.

Obtained funding: A. W. Dromerick, R. J. Holley, P. S. Lum.

Administrative, material, or technical support: R. J. Holley, B. Monroe, P. S. Lum.

Study supervision: A. W. Dromerick, P. S. Lum.

Financial Disclosures: The authors have declared that no competing interests exist.

Funding/Support: This material was based on work supported by the U.S. Army Medical Research and Materiel Command (contract W81XWH-05-1-0160).

Institutional Review: MedStar Health Research Institute (Hyattsville, Maryland) provided institutional review board approval for this study. We obtained informed consent from all subjects before participation.

Participant Follow-Up: The authors do not plan to inform participants of the publication of this study. However, participants have been encouraged to check the study Web site for updated publications.

\section{REFERENCES}

1. Ziegler-Graham K, MacKenzie EJ, Ephraim PL, Travison TG, Brookmeyer R. Estimating the prevalence of limb loss in the United States: 2005 to 2050. Arch Phys Med Rehabil. 2008;89(3):422-29. [PMID: 18295618]

DOI:10.1016/j.apmr.2007.11.005

2. Wright TW, Hagen AD, Wood MB. Prosthetic usage in major upper extremity amputations. J Hand Surg Am. 1995; 20(4):619-22. [PMID: 7594289$]$ DOI:10.1016/S0363-5023(05)80278-3

3. Dudkiewicz I, Gabrielov R, Seiv-Ner I, Zelig G, Heim M. Evaluation of prosthetic usage in upper limb amputees. Disabil Rehabil. 2004;26(1):60-63. [PMID: 14660200] DOI:10.1080/09638280410001645094

4. Dromerick AW, Schabowsky CN, Holley RJ, Monroe B, Markotic A, Lum P. Effect of training on upper-extremity prosthetic performance and motor learning: A single-case study. Arch Phys Med Rehabil. 2008;89(6):1199-1204.

[PMID: 18503820]

DOI:10.1016/j.apmr.2007.09.058

5. Graziano MS. Where is my arm? The relative role of vision and proprioception in the neuronal representation of limb position. Proc Natl Acad Sci U S A. 1999;96(18):10418-21. [PMID: 10468623]

DOI:10.1073/pnas.96.18.10418

6. Van Beers RJ, Wolpert DM, Haggard P. When feeling is more important than seeing in sensorimotor adaptation. Curr Biol. 2002;12(10):834-37. [PMID: 12015120] DOI:10.1016/S0960-9822(02)00836-9 
7. Sober SJ, Sabes PN. Flexible strategies for sensory integration during motor planning. Nat Neurosci. 2005;8(4):490-97. [PMID: 15793578]

8. Bagesteiro LB, Sainburg RL. Handedness: Dominant arm advantages in control of limb dynamics. J Neurophysiol. 2002;88(5):2408-21. [PMID: 12424282]

DOI:10.1152/jn.00901.2001

9. Shadmehr R, Mussa-Ivaldi FA. Adaptive representation of dynamics during learning of a motor task. J Neurosci. 1994;14(5 Pt 2):3208-24. [PMID: 8182467]

10. Schabowsky CN, Dromerick AW, Holley RJ, Monroe B, Lum PS. Trans-radial upper extremity amputees are capable of adapting to a novel dynamic environment. Exp Brain Res. 2008;188(4):589-601. [PMID: 18443766] DOI:10.1007/s00221-008-1394-9

11. Winter DA. Biomechanics and motor control of human movement. 2nd ed. New York (NY): Wiley; 1990.

12. Mathiowetz V, Weber K, Kashman N, Volland G. Adult norms for the Nine Hole Peg Test of finger dexterity. Am J Occup Ther. 1985;5(1):24-38.

13. Lyle RC. A performance test for assessment of upper limb function in physical rehabilitation treatment and research. Int J Rehabil Res. 1981;4(4):483-92. [PMID: 7333761] DOI:10.1097/00004356-198112000-00001

14. Scheidt RA, Rymer WZ. Control strategies for the transition from multijoint to single-joint arm movements studied using a simple mechanical constraint. J Neurophysiol. 2000;83(1): 1-12. [PMID: 10634848]

15. Lackner JR, Dizio P. Rapid adaptation to Coriolis force perturbations of arm trajectory. J Neurophysiol. 1994; 72(1):299-313. [PMID: 7965013]

16. Simpson DC. The choice of control system for the multimovement prosthesis: Extended physiological proprioception (EPP). In: Herberts P, Kadefors R, Magnusson R, Petersen I, editors. The control of upper-extremity prostheses and orthoses. Springfield (IL): Charles Thomas; 1974. p. 146-50.

17. Doeringer JA, Hogan N. Performance of above elbow body-powered prostheses in visually guided unconstrained motion tasks. IEEE Trans Biomed Eng. 1995;42(6):621-31. [PMID: 7790019]

DOI:10.1109/10.387202

18. Weeks DL, Wallace SA, Notebloom JT. Precision-grip force changes in the anatomical and prosthetic limb during predictable load increases. Exp Brain Res. 2000;132(3): 404-10. [PMID: 10883390]

DOI:10.1007/s002210000337

19. Criscimagna-Hemminger SE, Donchin O, Gazzaniga MS, Shadmehr R. Learned dynamics of reaching movements generalize from dominant to nondominant arm. J Neurophysiol. 2003;89(1):168-76. [PMID: 12522169] DOI:10.1152/jn.00622.2002
20. Marzi CA, Bisiacchi P, Nicoletti R. Is interhemispheric transfer of visuomotor information asymmetric? Evidence from a meta-analysis. Neuropsychologia. 1991;29(12): 1163-77. [PMID: 1838793] DOI:10.1016/0028-3932(91)90031-3

21. Morton SM, Lang CE, Bastian AJ. Inter- and intra-limb generalization of adaptation during catching. Exp Brain Res. 2001;141(4):438-45. [PMID: 11810138] DOI:10.1007/s002210100889

22. Thut G, Cook ND, Regard M, Leenders KL, Halsband U, Landis T. Intermanual transfer of proximal and distal motor engrams in humans. Exp Brain Res. 1996;108(2):321-27. [PMID: 8815040] DOI:10.1007/BF00228105

23. Wang J, Sainburg RL. Interlimb transfer of novel inertial dynamics is asymmetrical. J Neurophysiol. 2004;92(1):34960.

[PMID: 15028745]

DOI:10.1152/jn.00960.2003

24. Schaefer SY, Haaland KY, Sainburg RL. Ipsilesional motor deficits following stroke reflect hemispheric specializations for movement control. Brain. 2007;130(8):2146-58. [PMID: 17626039] DOI:10.1093/brain/awm145

25. Cohen LG, Bandinelli S, Findley TW, Hallett M. Motor reorganization after upper limb amputation in man. A study with focal magnetic stimulation. Brain. 1991;114(Pt 1B): 615-27. [PMID: 2004259] DOI:10.1093/brain/114.1.615

26. Kew JJ, Ridding MC, Rothwell JC, Passingham RE, Leigh PN, Sooriakumaran S, Frackowiak RS, Brooks DJ. Reorganization of cortical blood flow and transcranial magnetic stimulation maps in human subjects after upper limb amputation. J Neurophysiol. 1994;72(5):2517-24. [PMID: 7884476$]$

27. Röricht S, Meyer BU, Niehaus L, Brandt SA. Long-term reorganization of motor cortex outputs after arm amputation. Neurology. 1999;53(1):106-11. [PMID: 10408544]

28. Cramer SC, Nelles G, Benson RR, Kaplan JD, Parker RA, Kwong KK, Kennedy DN, Finklestein SP, Rosen BR. A functional MRI study of subjects recovered from hemiparetic stroke. Stroke. 1997;28(12):2518-27. [PMID: 9412643]

29. Rossini PM, Altamura C, Ferreri F, Melgari JM, Tecchio F, Tombini M, Pasqualetti P, Vernieri F. Neuroimaging experimental studies on brain plasticity in recovery from stroke. Eura Medicophys. 2007;43(2):241-54. [PMID: 17589415]

30. Yelnik A, Bonan I, Debray M, Lo E, Gelbert F, Bussel B. Changes in the execution of a complex manual task after ipsilateral ischemic cerebral hemispheric stroke. Arch Phys Med Rehabil. 1996;77(8):806-10. [PMID: 8702376] DOI:10.1016/S0003-9993(96)90261-0

31. Sunderland A, Bowers MP, Sluman SM, Wilcock DJ, Ardron ME. Impaired dexterity of the ipsilateral hand after 
stroke and the relationship to cognitive deficit. Stroke. 1999;30(5):949-55. [PMID: 10229726]

32. Yarosh CA, Hoffman DS, Strick PL. Deficits in movements of the wrist ipsilateral to a stroke in hemiparetic subjects. J Neurophysiol. 2004;92(6):3276-85. [PMID: 15295013] DOI:10.1152/jn.00549.2004

33. Wetter S, Poole JL, Haaland KY. Functional implications of ipsilesional motor deficits after unilateral stroke. Arch Phys Med Rehabil. 2005;86(4):776-81. [PMID: 15827931] DOI:10.1016/j.apmr.2004.08.009

34. Ferbert A, Priori A, Rothwell JC, Day BL, Colebatch JG, Marsden CD. Interhemispheric inhibition of the human motor cortex. J Physiol. 1992;453:525-46. [PMID: 1464843]
35. Sainburg RL. Evidence for a dynamic-dominance hypothesis of handedness. Exp Brain Res. 2002;142(2):241-58. [PMID: 11807578]

DOI:10.1007/s00221-001-0913-8

36. Sainburg RL. Handedness: Differential specializations for control of trajectory and position. Exerc Sport Sci Rev. 2005;33(4):206-13. [PMID: 16239839] DOI:10.1097/00003677-200510000-00010

Submitted for publication June 9, 2009. Accepted in revised form December 29, 2009. 
